\title{
Sevoflurane exerts a more marked influence compared with propofol on gene expression in patients undergoing coronary artery bypass graft surgery
}

\author{
HUA $\mathrm{LI}^{1,2}$, JING CANG ${ }^{1,2}$ and XIAOGUANG ZHANG ${ }^{1,2}$ \\ Departments of ${ }^{1}$ Anesthesia and ${ }^{2}$ Anesthesiology, Zhongshan Hospital, Shanghai Medical College, \\ Fudan University, Shanghai 200032, P.R. China
}

Received October 22, 2014; Accepted October 22, 2015

DOI: $10.3892 / \mathrm{etm} .2015 .2936$

\begin{abstract}
The aim of the present study was to elucidate the influence of the anesthetics propofol and sevoflurane on gene expression in patients undergoing coronary artery bypass graft surgery (CABG) and to provide a basis for the selection of the appropriate anesthetic. The gene expression profiles of patients receiving one of the two anesthetics were analyzed prior to and following the induction of anesthesia. GSE4386 microarray data obtained from the Gene Expression Omnibus database was used to identify the differentially expressed genes (DEGs) by significance analysis of the microarray. The data set contained data regarding atrial tissue samples from 40 patients that underwent $\mathrm{CABG}$, and that received either propofol $(n=10)$ or sevoflurane $(n=10)$ or were control subjects $(n=20)$. The 20 control samples comprised the same patients prior to undergoing CABG. The Kyoto Encyclopedia of Genes and Genomes and Gene Ontology (GO) Enrichment Analysis was applied to the DEGs using the Database for Annotation, Visualization and Integration Discovery functional annotation bioinformatics microarray tool. A total of 242 and 560 DEGs were identified in the human atrial samples treated with propofol and sevoflurane, respectively. Among these, 116 upregulated DEGs and no downregulated DEGs were found to be unique to sevoflurane, while 10 upregulated and 212 downregulated DEGs were unique to propofol. The majority of the pathways that were significantly over-represented among the upregulated DEGs were associated with the immune response, such as Toll- and NOD-like
\end{abstract}

Correspondence to: Dr Jing Cang or Dr Xiaoguang Zhang, Department of Anesthesia, Zhongshan Hospital, Shanghai Medical College, Fudan University, 180 Fenglin Road, Shanghai 200032, P.R. China

E-mail: jingcangjc@163.com

E-mail: xiaoguangzhangxgz@163.com

Key words: coronary artery bypass graft, sevoflurane, propofol, gene expression profile, differentially expressed genes, functional enrichment analysis, gene ontology receptors and Jak-STAT signaling pathways. GO enrichment analysis revealed that the downregulated DEGs unique to sevoflurane treatment were involved in the immune response and glucose metabolism, while the upregulated DEGs were associated with cellular ion homeostasis and epithelial cell development. Compared with propofol, sevoflurane appeared to exert a more marked effect on biological pathways, such as drug metabolism, glycolysis, cellular ion homeostasis and epithelial cell development.

\section{Introduction}

Coronary artery bypass grafting (CABG) has been recognized as one of the most efficacious methods for treating coronary heart disease (1). Sevoflurane and propofol are commonly used anesthetics in CABG surgery $(2,3)$.

Propofol has a chemical structure that is similar to that of antioxidants, and has been shown to be able to scavenge free radicals in vivo (4). Previous studies have shown that propofol exerts different effects on various receptors and ion channels of the central nervous system $(5,6)$. Propofol is able to reduce $\beta$-adrenoreceptor-mediated signal transduction in cardiomyocytes, via a protein kinase C-dependent pathway. Furthermore, Sayin et al (7) proposed that propofol is able to attenuate myocardial lipid peroxidation during CAGB surgery. Corcoran et al (8) further suggested that propofol decreases free radical-mediated lipid peroxidation and the systemic inflammatory response in patients undergoing CAGB surgery.

Sevoflurane has been shown to serve a protective function in the pharmacological preconditioning of cardiac events in patients undergoing CABG $(9,10)$. Furthermore, sevoflurane reduces the incidence of late cardiac events during the first year following CABG surgery, which may occur by downregulating the expression of platelet endothelial cell adhesion molecule-1 (9). Yao et al (11) proposed that the myocardial protection exerted by sevoflurane in CABG surgery is achieved through the downregulation of troponin I. In addition, previous meta-analyses have further confirmed the protective effect of sevoflurane in cardiac surgery $(12,13)$.

Numerous studies have compared the myocardial protective effects of sevoflurane and propofol in patients undergoing CABG surgery, and the relative advantages and disadvantages 
of the two methods have been presented $(14,15)$. The use of sevoflurane appears to result in superior outcome compared with propofol in patients with little or no indication of ischemic heart disease, including patients undergoing $\mathrm{CABG}$ surgery (16). However, sevoflurane exhibits more marked antioxidative properties compared with propofol in patients undergoing off-pump CABG, as indicated by the results of a randomized controlled study (17). Furthermore, sevoflurane has been shown to possess stronger myocardial protective effects compared with propofol in patients undergoing $\mathrm{CABG}$ surgery (18). However, the underlying mechanisms of this protective effect remain unclear.

Microarray technology enables the global determination of gene expression levels, and is thus useful for the elucidation of underlying molecular mechanisms. Therefore, microarray analysis may useful for determining the effects of sevoflurane and propofol on gene expression in patients undergoing CABG. Lucchinetti et al (19) suggested that gene regulatory control of myocardial energy metabolism is closely associated with postoperative cardiac function. In the present study, a data set from the Gene Expression Omnibus (GEO) database (GSE4386; http://www.ncbi.nlm.nih.gov/geo/query/acc. cgi?acc=GSE4386) was downloaded and was subjected to screening for differentially expressed genes (DEGs). The aim of this study was to determine the influence of propofol and sevoflurane on postoperative recovery in patients following CABG. Gene Ontology (GO) and Kyoto Encyclopedia of Genes and Genomes (KEGG) pathway enrichment analyses were performed to indicate the underlying molecular mechanisms of any effects and identify potential biomarkers. Such markers may facilitate the appropriate selection of sevoflurane or propofol, thereby improving the outcomes of patients undergoing $\mathrm{CABG}$.

\section{Materials and methods}

Gene expression data. The microarray data set GSE4386 was downloaded from the platform of GeneChip ${ }^{\circledR}$ Human Genome U133 Plus 2.0 Array of the GEO database (http://www.ncbi. nlm.nih.gov/geo/) (20). The data from a total of 40 samples was contained in the data set, including data from patients undergoing CABG surgery combined with sevoflurane treatment $(n=10)$, propofol treatment $(n=10)$ and control samples $(n=20)$. The control samples comprised the same patients prior to CABG surgery. Atrial samples were collected prior to and following CABG surgery to determine gene expression profiles. The patients were treated in Triemli Hospital (Zurich, Switzerland). The mean ages of patients in the propofol and sevoflurane groups were 66.9 and 65.2 years, respectively. All patients were male. In addition, patients with hemodynamic instability was excluded. Microarray analysis was performed based on GSE4386, in which total RNA was prepared from the frozen cardiac tissue using an RNeasy Mini kit (Qiagen, Hilden, Germany).

Pretreatment of raw data and DEGs analysis. Raw data were processed using Log2 transformation and quantile normalization, using SPSS software, version 17.0 (SPSS, Inc., Chicago, IL, USA). The mRNA expression level was calculated based on the annotation files of probes. Gene expression profiles
Table I. Differentially expressed mRNA sequences and genes in propofol and sevoflurane.

\begin{tabular}{lcccc}
\hline Parameter & P_up & P_down & S_up & S_down \\
\hline mRNAs (n) & 353 & 41 & 558 & 408 \\
Genes (n) & 217 & 25 & 323 & 237 \\
\hline
\end{tabular}

P, propofol; S, sevoflurane; $\mathrm{P} \_$up, upregulated genes in $\mathrm{P}$; $\mathrm{P} \_$down, downregulated genes in P; S_up, upregulated genes in S; S_down, downregulated genes in $\mathrm{S}$.

prior to and following anaesthesia with sevoflurane or propofol were compared using the $\mathrm{R}$ statistical program in a Limma software (21). The threshold of DEGs was considered to be $\mathrm{P}<0.05$ and $\log 2$ (fold-change) of $>1$.

Functional enrichment analysis. The database for Annotation, Visualization and Integration Discovery (DAVID; http://david.abcc.ncifcrf.gov/) (22) provides analytical tools for analyzing a large list of genes, and was used to perform GO (http://geneontology.org/page/go-enrichment-analysis) and KEGG (http://www.genome.jp/kegg/) pathway enrichment analyses for DEGs. $\mathrm{P}<0.05$ was considered to indicate a statistically significant difference.

\section{Results}

Sevoflurane influences more genes compared with propofol at the transcriptional level. A total of 34,296 mRNA sequences (corresponding to 19,745 genes) were identified following raw data pretreatment. Compared with the control samples, 242 and 560 DEGs were detected in patients treated with propofol and sevoflurane, respectively (Table I). Following the comparison between the DEGs identified in the two treatment groups, 207 upregulated and 25 downregulated DEGs were found to overlap (Fig. 1). By contrast, 116 upregulated and no downregulated DEGs were unique to sevoflurane, while 10 upregulated and 212 downregulated DEGs were unique to propofol.

GO term and KEGG pathway enrichment analyses. Significantly enriched KEGG pathways and GO terms, and their enriched DEGs, are presented in Table II. A total of 10 KEGG pathways were enriched by 207 upregulated DEGs, while these DEGs enriched into 243 GO terms. As shown in Fig. 2, the majority of the pathways of upregulated overlapping DEGs were associated with immune responses, such as Toll-like receptor (TLR), NOD-like receptor (NLR), Jak-STAT and MAPK signaling pathways, in addition to cytokine-cytokine receptor interaction. The KEGG enrichment results for downregulated DEGs associated with sevoflurane are presented in Fig. 3. A total of three pathways were associated, including complement and coagulation cascades, glycolysis and drug metabolism. The pathway associated with cytokine-cytokine receptor interaction was found to be uniquely downregulated by sevoflurane.

As shown in Fig. 4, the GO terms which were found to be significantly enriched in the 212 downregulated genes 
Table II. Numbers of significantly enriched KEGG pathways and GO terms.

\begin{tabular}{lcccccccccc}
\hline Parameter & $\begin{array}{c}\text { Up } \\
\text { common }\end{array}$ & P_up & S_up & $\begin{array}{c}\text { P_up } \\
\text { _uniq }\end{array}$ & $\begin{array}{c}\text { S_up } \\
\text { _uniq }\end{array}$ & $\begin{array}{c}\text { down_ } \\
\text { common }\end{array}$ & $\begin{array}{c}\text { P_c }_{-} \\
\text {down }\end{array}$ & $\begin{array}{c}\text { S_ } \\
\text { down }\end{array}$ & $\begin{array}{c}\text { P_down } \\
\text { _uniq }\end{array}$ & $\begin{array}{c}\text { S_down } \\
\text { _uniq }\end{array}$ \\
\hline Gene (n) & 207 & 217 & 323 & 10 & 116 & 25 & 25 & 237 & 0 & 212 \\
KEGG pathway (n) & 10 & 10 & 13 & 1 & 1 & 0 & 0 & 3 & 0 & 4 \\
GO biological pathway (n) & 243 & 250 & 321 & 4 & 95 & 0 & 0 & 49 & 0 & 50 \\
\hline
\end{tabular}

P, propofol; S, sevoflurane; Up_common, upregulated genes common between P and S; P_up, upregulated genes in P; S_up, upregulated genes in $\mathrm{S}$; P_up_uniq, upregulated genes unique to P; S_up_uniq, upregulated genes unique to $\mathrm{S}$; Down_common, downregulated genes common between P and S; P_down, downregulated genes in P; S_down, downregulated genes in S; P_down_uniq, downregulated genes unique to P; S_down_uniq, downregulated genes unique to S. GO, Gene Ontology; KEGG, Kyoto Encyclopedia of Genes and Genomes.
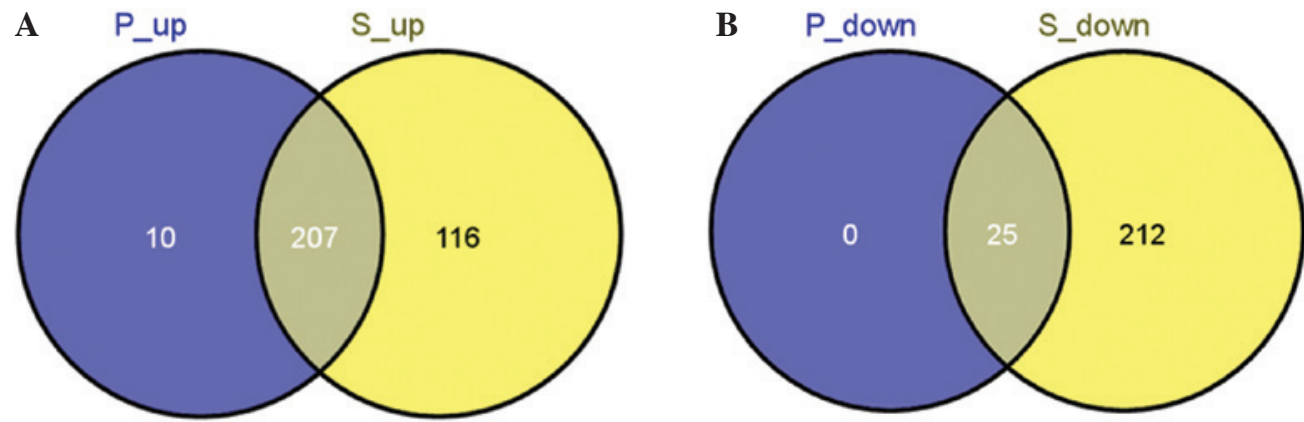

Figure 1. Venn diagram showing the number of (A) upregulated and (B) downregulated genes between P and S. P, propofol; S, sevoflurane; $P_{-}$up, upregulated genes in P; P_down, downregulated genes in P; S_up, upregulated genes in S; S_down, downregulated genes in S.

that were unique to sevoflurane could be divided into three groups: Immune response, glucose metabolism and response to vitamin and nutrient.

The GO biological pathways, which were found to be significantly over-represented in the 116 upregulated genes that were unique to sevoflurane could be divided into seven groups, including pathways associated with the immune response, apoptosis, cellular ion homeostasis and epithelial cell development. Among these pathways, those associated with cellular ion homeostasis (Fig. 5A) and epithelial cell development (Fig. 5B) were not detected among the pathways enriched by DEGs commonly upregulated by propofol and sevoflurane.

\section{Discussion}

Sevoflurane and propofol are the two most commonly used anesthetics in CABG surgery (23). In the present study, a CABG patient data set (GSE4386) was downloaded from the GEO database to determine the molecular mechanisms underlying the effects of the two anesthetics. A total of 242 and 560 DEGs were identified in patients treated with propofol and sevoflurane, respectively. The two groups were found to have 207 downregulated DEGs in common, 116 that were unique to sevoflurane and 10 that were unique to propofol. In total, 25 downregulated genes were shared and 212 were unique to sevoflurane, while none was unique to propofol. The upregulated DEGs unique to sevoflurane were associated with cellular ion homeostasis and epithelial cell development, while the unique downregulated genes were associated with glucose metabolism, immune response and response to vitamin.
In the present study, pathways associated with cellular ion homeostasis, such as the regulation of membrane potential, cellular metal ion homeostasis and cellular calcium ion homeostasis, were enriched by upregulated DEGs unique to sevoflurane. Sevoflurane anesthesia has previously been shown to alter the electrophysiological activity of neurons by reducing hypoxic depolarization and enhancing the hypoxic hyperpolarization, thus protecting neurons against ischemia (24). Furthermore, sevoflurane has been found to increase coronary collateral blood flow through the activation of calcium-activated potassium channels (25). In the present study, the upregulated DEGs that were unique to sevoflurane were additionally associated with epithelial cell development, such as tissue morphogenesis, and epithelium and blood vessel development. Previously, sevoflurane treatment was shown to increase the synthesis of heat shock protein (HSP)-70 without affecting HSP-32 and HSP-27 synthesis (26). In the process of ischemia and reperfusion, HSP-70 was able to protect the heart through the expression of CD69 and by inducing a reduction in intracellular calcium (27). Sevoflurane may therefore serve a crucial function in mediating cardioprotection by influencing the pathways associated with cellular ion homeostasis and epithelial cell development.

In addition, unique downregulated DEGs associated with sevoflurane anesthesia were enriched in the pathways associated with glucose metabolism, immune response and response to vitamin in the present study. Saho et al (28) previously found that sevoflurane anesthesia may reversibly inhibit basal and glucose-stimulated insulin secretion, and further induce insulin resistance. Insulin resistance has been identified as 


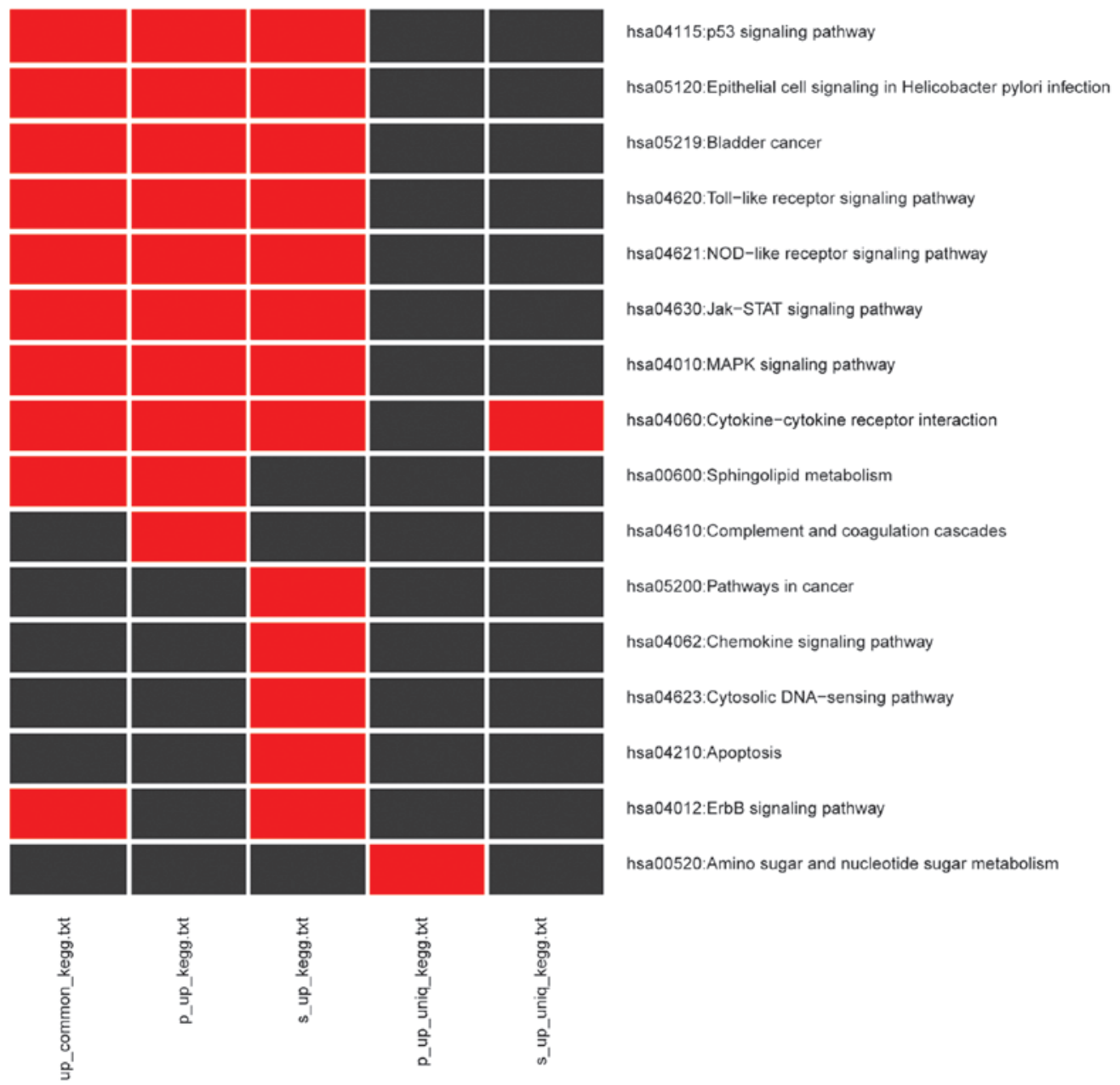

Figure 2. KEGG pathways enriched in upregulated genes. Significant pathways are red while insignificant pathways are black. KEGG, Kyoto Encyclopedia of Genes and Genomes. up_common_kegg, KEGG pathways enriched in common upregulated genes; p_up_kegg, KEGG pathways enriched in upregulated genes of propofol; s_up_kegg, KEGG pathways enriched in upregulated genes of sevoflurane; p_up_uniq_kegg. KEGG pathways enriched in unique upregulated genes of propofol; s_up_uniq_kegg, KEGG pathways enriched in unique upregulated genes of sevoflurane.

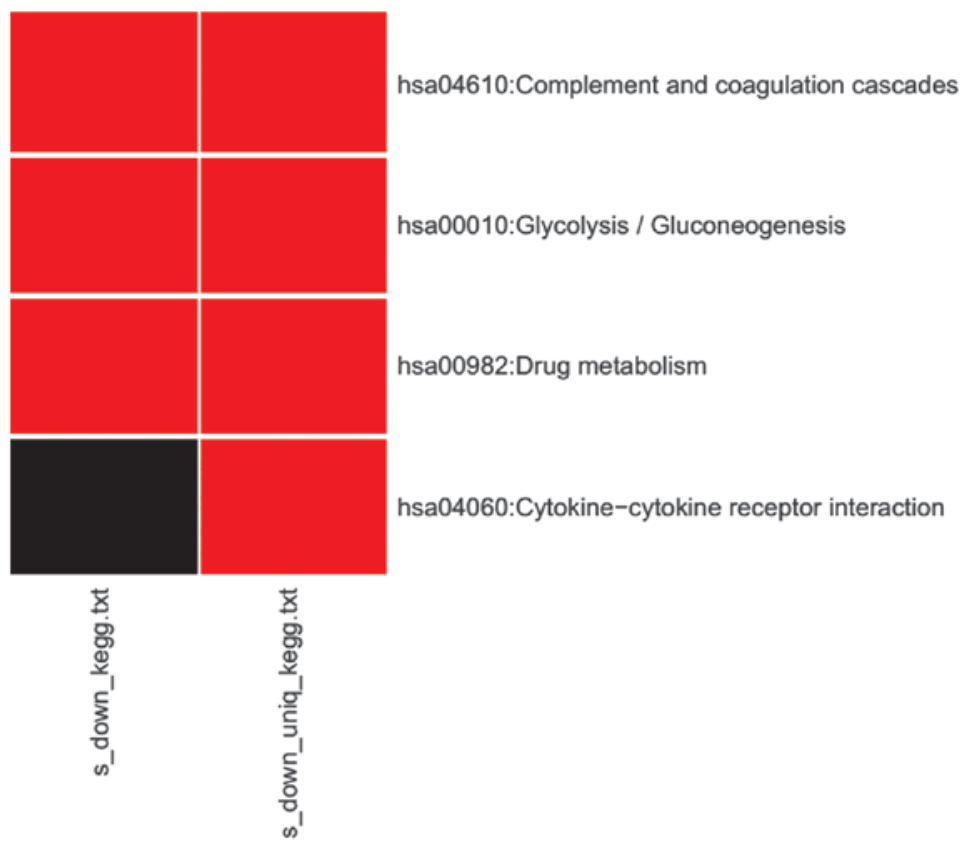

Figure 3. KEGG pathways enriched downregulated genes. Significant pathways are red while insignificant pathways are black. KEGG, Kyoto Encyclopedia of Genes and Genomes. s_down_kegg, pathways enriched in downregulated genes of sevoflurane; s_down_uniq_kegg, KEGG pathways enriched in unique downregulated genes of sevoflurane. 


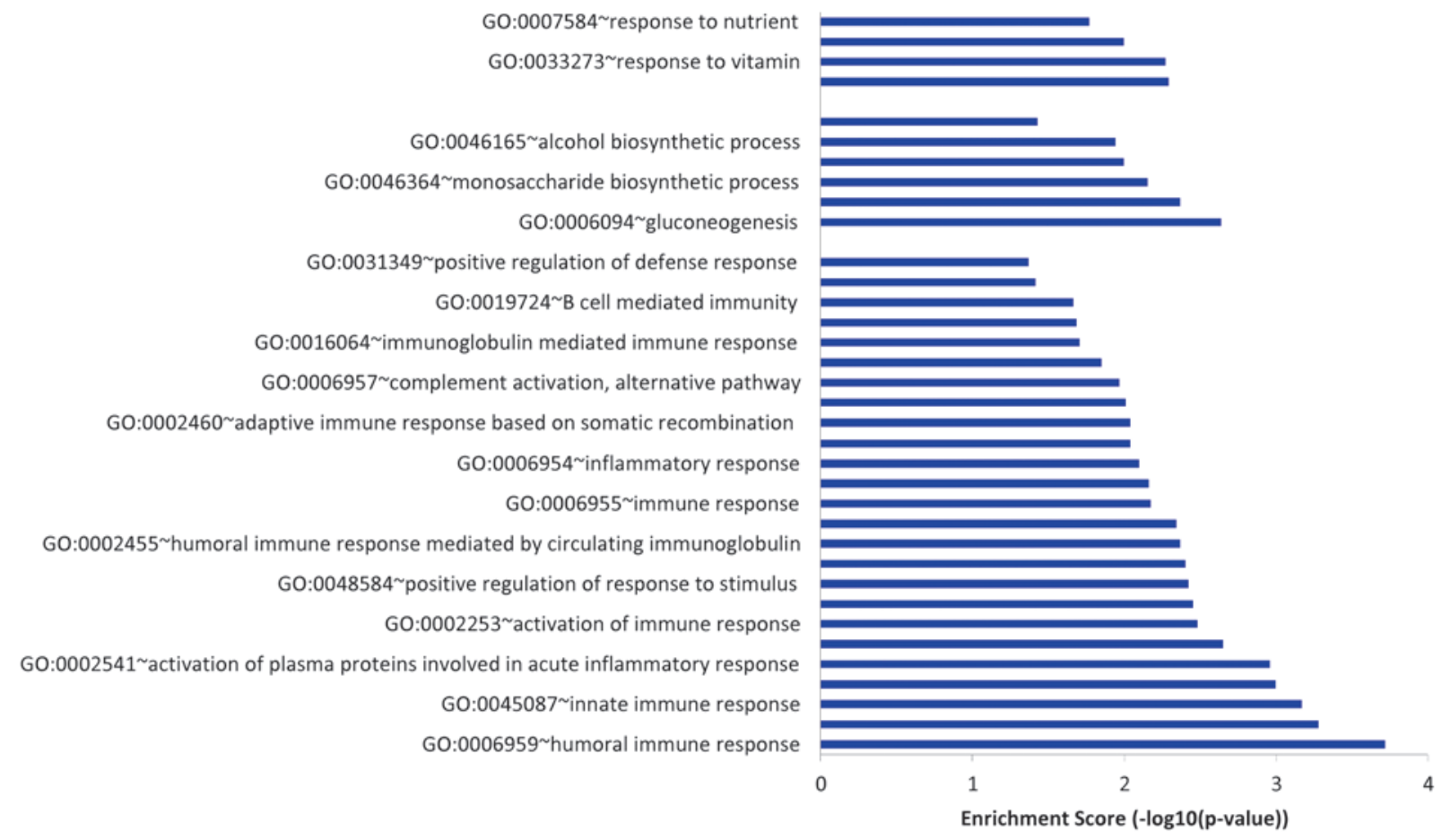

Figure 4. GO biological pathway terms significantly enriched in the 212 downregulated genes unique to patients that received sevoflurane. GO, Gene Ontology.

A

GO:0042391 regulation of membrane potential

GO:0006875 cellular metal ion homeostasis

GO:0006874 cellular calcium ion homeostasis

GO:0030003 cellular cation homeostasis

GO:0019725 cellular homeostasis

GO:0048878 chemical homeostasis

GO:0055066 di-, tri-valent inorganic cation homeostasis

GO:0006873 cellular ion homeostasis

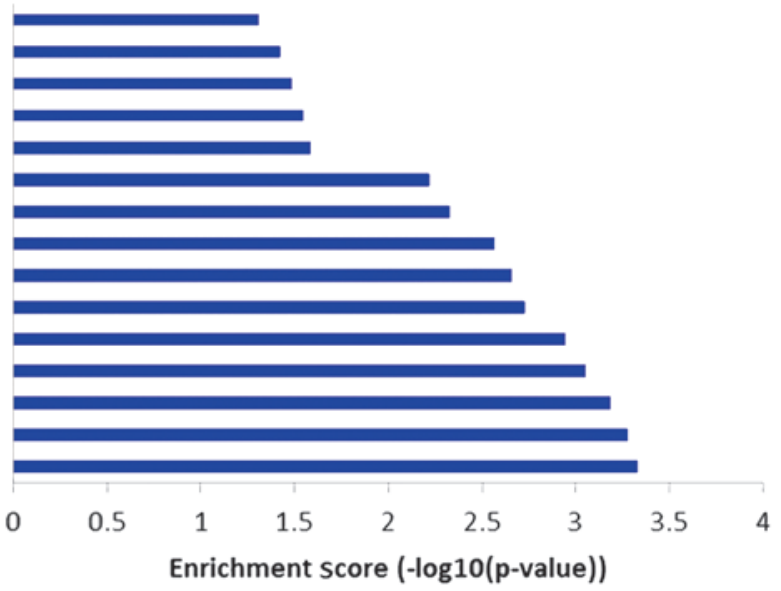

B

GO:0001654 eye development GO:0035295 tube development

GO:0035239 tube morphogenesis

GO:0001655 urogenital system development

GO:0001656 metanephros development

GO:0043010 camera-type eye development

GO:0048729 tissue morphogenesis

GO:0002009 morphogenesis of an epithelium

GO:0001822 kidney development

GO:0001657 ureteric bud development

GO:0060429 epithelium development

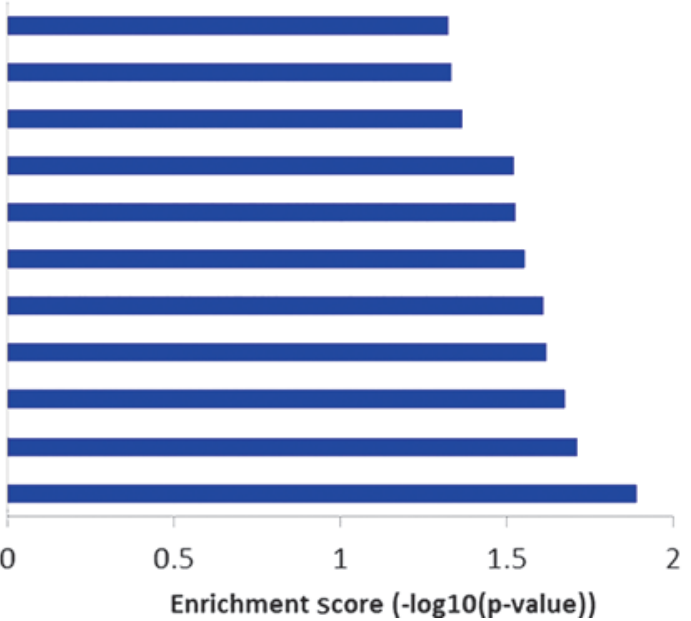

Figure 5. GO biological pathway terms significantly over-represented in the 116 genes upregulated in sevoflurane alone. (A) Cluster 3, pathways associated with cellular ion homeostasis. (B) Cluster 7, pathways associated with epithelial cell development. GO, Gene Ontology. 
an independent risk factor for coronary heart disease (29). Therefore, understanding the glycometabolic state of patients prior to CABG surgery is crucial in order to reduce the postoperative complications.

In the present study, these upregulated DEGs were enriched in pathways associated with the immune response, including innate, immunoglobulin-mediated and humoral immune responses; however, these upregulated pathways were additionally associated with the physical injury caused by CABG surgery, and thus were shared between sevoflurane and propofol. During the process of the immune response, TLRs have been confirmed as the signaling receptor for HSPs, mediating the synthesis of inflammatory cytokines (30). In addition, NLRs, TLRs and RIG-1-like receptors have been found to be involved in immune responses (31-36), and response to vitamin was shown to be a key pathway enriched by downregulated DEGs associated with sevoflurane anesthesia by the present results. Samadikhah et al (37) found that vitamin $\mathrm{C}$ combined with oral atorvastatin was significantly effective at preventing post-CABG atrial fibrillation. Furthermore, vitamin D, which is activated by a low-calcemic agonist, has been shown to modulate the humoral immune response, further affecting the efficacy of the surgical outcome of CABG (38). Collectively, these results suggest that sevoflurane exhibits a more marked effect on biological pathways compared with propofol, but exhibits a number of shortcomings. In order to achieve a more beneficial use of sevoflurane anesthesia in patients undergoing $\mathrm{CABG}$, certain complementary therapies, such as the regulation of glucose balance and the use of vitamin supplements, should be considered.

In conclusion, the pathways enriched by DEGs, particularly those that were unique to sevoflurane and propofol, may affect surgical outcomes in patients undergoing CABG. In the present study, sevoflurane exhibited a more marked impact on the biological pathways investigated compared with propofol; however, the identified DEGs and pathways in this study were not investigated in animal models. Further study of this subject in animal models may be required in the future.

\section{Acknowledgements}

This study was supported by the Shanghai Natural Science Foundation Project (grant no. 14ZR1406900). The authors thank Dr Hao Wang of the Departments of Anesthesia and Anesthesiology (Shanghai Medical College, Fudan University, Shanghai, China) for assistance with data analysis and valuable discussion.

\section{References}

1. Møller C, Penninga L, Wetterslev J, Steinbrüchel D and Gluud C: Off-pump versus on-pump coronary artery by-pass grafting for ischaemic heart disease. Cochrane Database Syst Rev 3: CD007224, 2012.

2. Wang J, Zheng H, Chen CL, Lu W and Zhang YQ: Sevoflurane at 1 MAC provides optimal myocardial protection during off-pump CABG. Scand Cardiovasc J 47: 175-184, 2013.

3. Hellström J, Öwall A, Bergström J and Sackey P: Cardiac outcome after sevoflurane versus propofol sedation following coronary bypass surgery: A pilot study. Acta Anaesthesiol Scand 55: 460-467, 2011.
4. Gülçin I, Alici HA and Cesur M: Determination of in vitro antioxidant and radical scavenging activities of propofol. Chem Pharm Bull (Tokyo) 53: 281-285, 2005.

5. Marik PE: Propofol: Therapeutic indications and side-effects. Curr Pharm Des 10: 3639-3649, 2004.

6. Vakkuri A, Yli-Hankala A, Talja P,Mustola S, Tolvanen-LaaksoH, Sampson T and Viertiö-Oja H: Time-frequency balanced spectral entropy as a measure of anesthetic drug effect in central nervous system during sevoflurane, propofol and thiopental anesthesia. Acta Anaesthesiol Scand 48: 145-153, 2004.

7. Sayin MM, Özatamer O, Taşöz R, Kilinc K and Ünal N: Propofol attenuates myocardial lipid peroxidation during coronary artery bypass grafting surgery. Br J Anaesth 89: 242-246, 2002.

8. Corcoran TB, Engel A, Sakamoto H, O'Shea A O'Callaghan-Enright S and Shorten G: The effects of propofol on neutrophil function, lipid peroxidation and inflammatory response during elective coronary artery bypass grafting in patients with impaired ventricular function. Br J Anaesth 97: 825-831, 2006

9. Garcia C, Julier K, Bestmann L, Zollinger A, von Segesser LK, Pasch T, Spahn DR and Zaugg M: Preconditioning with sevoflurane decreases PECAM-1 expression and improves one-year cardiovascular outcome in coronary artery bypass graft surgery. Br J Anaesth 94: 159-165, 2005.

10. Lucchinetti E, da Silva R, Pasch T, Schaub MC and Zaugg M: Anaesthetic preconditioning but not postconditioning prevents early activation of the deleterious cardiac remodelling programme: Evidence of opposing genomic responses in cardioprotection by pre-and postconditioning. $\mathrm{Br} \mathrm{J}$ Anaesth 95: 140-152, 2005

11. Yao YT and Li LH: Sevoflurane versus propofol for myocardial protection in patients undergoing coronary artery bypass grafting surgery: A meta-analysis of randomized controlled trials. Chin Med Sci J 24: 133-141, 2009.

12. Landoni G, Biondi-Zoccai GG, Zangrillo A, Bignami E, D'Avolio S, Marchetti C, Calabrò MG, Fochi O, Guarracino F, Tritapepe L, et al: Desflurane and sevoflurane in cardiac surgery: A meta-analysis of randomized clinical trials. J Cardiothorac Vasc Anesth 21: 502-511, 2007.

13. Yu CH and Beattie WS: The effects of volatile anesthetics on cardiac ischemic complications and mortality in CABG: A meta-analysis. Can J Anaesth 53: 906-918, 2006.

14. Yao YT and Li LH: Sevoflurane versus propofol for myocardial protection in patients undergoing coronary artery bypass grafting surgery: A meta-analysis of randomized controlled trials. Chin Med Sci J 24: 133-141, 2009.

15. Jakobsen CJ, Berg H, Hindsholm KB, Faddy N and Sloth E: The influence of propofol versus sevoflurane anesthesia on outcome in 10,535 cardiac surgical procedures. J Cardiothorac Vasc Anesth 21: 664-671, 2007.

16. Jakobsen CJ, Berg H, Hindsholm KB, Faddy N and Sloth E: The influence of propofol versus sevoflurane anesthesia on outcome in 10,535 cardiac surgical procedures. J Cardiothorac Vasc Anesth 21: 664-671, 2007.

17. Ballester M, Llorens J, Garcia-de-la-Asuncion J, Perez-Griera J, TebarE, Martinez-Leon J, Belda J and Juez M: Myocardial oxidative stress protection by sevoflurane vs. propofol: A randomised controlled study in patients undergoing off-pump coronary artery bypass graft surgery. Eur J Anaesthesiol 28: 874-881, 2011.

18. Yao YT and Li LH: Sevoflurane versus propofol for myocardial protection in patients undergoing coronary artery bypass grafting surgery: A meta-analysis of randomized controlled trials. Chin Med Sci J 24: 133-141, 2009.

19. Lucchinetti E, Hofer C, Bestmann L, Hersberger M, Feng J, Zhu M, Furrer L, Schaub MC, Tavakoli R, Genoni M, et al: Gene regulatory control of myocardial energy metabolism predicts postoperative cardiac function in patients undergoing off-pump coronary artery bypass graft surgery: Inhalational versus intravenous anesthetics. Anesthesiology 106: 444-457, 2007.

20. Barrett T, Suzek TO, Troup DB, Wilhite SE, Ngau WC, Ledoux P, Rudnev D, Lash AE, Fujibuchi W and Edgar R: NCBI GEO: Mining millions of expression profiles-database and tools. Nucleic Acids Res 33 (Database Issue): D562-D566, 2005.

21. Smyth GK: Linear models and empirical bayes methods for assessing differential expression in microarray experiments. Stat Appl Genet Mol Biol 3: Article3, 2004.

22. Dennis G Jr, Sherman BT, Hosack DA, Yang J, Gao W, Lane HC and Lempicki RA: DAVID: Database for annotation, visualization and integrated discovery. Genome biol 4: P3, 2003. 
23. Soro M, Gallego L, Silva V, Ballester MT, Lloréns J, Alvariño A García-Perez ML, Pastor E, Aguilar G, Martí FJ, et al: Cardioprotective effect of sevoflurane and propofol during anaesthesia and the postoperative period in coronary bypass graft surgery: a double-blind randomised study. Eur J Anaesthesiol 29: 561-569, 2012.

24. Wang J, Lei B, Popp S, Meng F, Cottrell J and Kass I: Sevoflurane immediate preconditioning alters hypoxic membrane potential changes in rat hippocampal slices and improves recovery of CA1 pyramidal cells after hypoxia and global cerebral ischemia. Neuroscience 145: 1097-1107, 2007.

25. Kehl F, Krolikowski JG, Tessmer JP, Pagel PS, Warltier DC and Kersten JR: Increases in coronary collateral blood flow produced by sevoflurane are mediated by calcium-activated potassium (BKCa) channels in vivo. Anesthesiology 97: 725-731, 2002.

26. Lee HT, Kim M, Jan M and Emala CW: Anti-inflammatory and antinecrotic effects of the volatile anesthetic sevoflurane in kidney proximal tubule cells. Am J Physiol Renal Physiol 291: F67-F78, 2006

27. Sharma M, Ganguly NK, Chaturvedi G, Thingnam SK, Majumdar S and Suri RK: A possible role of HSP70 in mediating cardioprotection in patients undergoing CABG. Mol Cell Biochem 247: 31-36, 2003.

28. Saho S, Kadota Y, Sameshima T, Miyao J, Tsurumaru T and Yoshimura N: The effects of sevoflurane anesthesia on insulin secretion and glucose metabolism in pigs. Anesth Analg 84: $1359-1365,1997$.

29. Ai M, Otokozawa S, Asztalos BF, White CC, Cupples LA Nakajima K, Lamon-Fava S, Wilson PW, Matsuzawa Y and Schaefer EJ: Adiponectin: An independent risk factor for coronary heart disease in men in the Framingham offspring study. Atherosclerosis 217: 543-548, 2011.
30. Dybdahl B, Wahba A, Lien E, Flo TH, Waage A, Qureshi N, Sellevold OF, Espevik T and Sundan A: Inflammatory response after open heart surgery: Release of heat-shock protein 70 and signaling through toll-like receptor-4. Circulation 105: 685-690, 2002.

31. Akira S and Takeda K: Toll-like receptor signalling. Nat Rev Immunol 4: 499-511, 2004.

32. Kanneganti TD, Lamkanfi M and Núñez G: Intracellular NOD-like receptors in host defense and disease. Immunity 27: 549-559, 2007.

33. Kaminska B: MAPK signalling pathways as molecular targets for anti-inflammatory therapy-from molecular mechanisms to therapeutic benefits. Biochim Biophys Acta 1754: 253-262, 2005.

34. Murray PJ: The JAK-STAT signaling pathway: Input and output integration. J Immunol 178: 2623-2629, 2007.

35. Appledorn DM, Patial S, McBride A, Godbehere S, Van Rooijen N, Parameswaran N and Amalfitano A: Adenovirus vector-induced innate inflammatory mediators, MAPK signaling, as well as adaptive immune responses are dependent upon both TLR2 and TLR9 in vivo. J Immunol 181: 2134-2144, 2008.

36. Huang G, Shi LZ and Chi H: Regulation of JNK and p38 MAPK in the immune system: Signal integration, propagation and termination. Cytokine 48: 161-169, 2009.

37. Samadikhah J, Golzari SE, Sabermarouf B, Karimzadeh I, Tizro P, Mohammad Khanli H and Ghabili K: Efficacy of combination therapy of statin and vitamin $\mathrm{C}$ in comparison with statin in the prevention of post-CABG atrial fibrillation. Adv Pharm Bull 4: 97-100, 2014

38. Baeke F, Takiishi T, Korf H, Gysemans C and Mathieu C: Vitamin D: Modulator of the immune system. Curr Opin Pharmacol 10: 482-496, 2010. 